sich auch nicht als eine besondere Ausführung der Computertomographie darstellen, sodass für sie daher nur eine Analogbewertung in Betracht kommen dürfte. Ob diese nun zutreffend schon mit der analogen Anwendung der Nrn. 5370 und 5377 erfasst ist, wie die Antragsgegnerin meint, oder darüber hinaus die Nrn. 5002, 5090, 5095, 5290 analog in Ansatz zu bringen sind, wie der Antragsteller fordert, lässt sich nicht ohne Einbeziehung wertender ärztlicher und rechtlicher Gesichtspunkte bestimmen. Aus ärztlicher Sicht hat der Antragsteller mit Blick auf die notwendige medizinische Indikation hierzu in seiner Stellungnahme v. 15.3.2005 umfassend vorgetragen. Welche rechtliche Bewertung letztlich zutreffend ist, kann dahinstehen. Eine relevante Berufspflichtverletzung, die eine Rüge zur Folge haben könnte, vermag hieraus jedenfalls nicht zu resultieren.

Letztlich sprechen auch verfassungsrechtliche Erwägungen dafür, nur erhebliche - hier aber nicht vorliegende Sorgfaltsverstöße bei der Erstellung einer ärztlichen Liquidation als berufsrechtlich relevant anzusehen. Es ist zwar grundsätzlich Sache des Verordnungsgebers der Gebührenordnung für Ärzte, darüber zu befinden, wie ärztliche Leistungen, ggf. auch unter Berücksichtigung nach Erlass der Verordnung eingetretener Veränderungen des technischen Standards oder der Fortentwicklung wissenschaftlicher Erkenntnisse, zu bewerten sind. Eine Bindung an die Verordnung besteht allerdings dann nicht, wenn sie wegen Verstoßes gegen höherrangiges Recht - etwa Art. 3 oder Art. 12 GG - nichtig ist (vgl. BGH, Urt. v. 18.9.2003 III ZR 389/02 -, NJW-RR 2003, 1639).

Es muss dem Arzt auch in Anbetracht seiner Berufsfreiheit zugestanden werden, sich auf behauptete Verletzungen seiner Grundrechte zu berufen, ohne zugleich berufsrechtliche Sanktionen fürchten zu müssen. Dies wäre aber der Fall, wenn ein Berufsrechtsverstoß bereits dann vorläge, wenn der Arzt etwa mit vertretbarer Begründung vorträgt, die Gebührenordnung für Ärzte trage nach ihrem Erlass eingetretenen Veränderungen nicht hinreichend Rechnung, seine Auffassung aber im Nachhinein keine (zivil)gerichtliche Billigung findet.

\title{
REZENSIONEN
}

DOI: 10.1007/s00350-011-2935-y

\section{Vertragsärztlicher Abrechnungsbetrug und Schadensbestimmung.}

Von Caspar Luig. (Europäische Hochschulschriften, Bd. 4928), Verlag Peter Lang, Frankfurt a.M. 2009, 263 S., kart., €52,80

Die bereits im Jahr 2009 in der Reihe der Europäischen Hochschulschriften veröffentlichte Arbeit von Luig hat bislang im medizinrechtlichen Schrifttum nur vereinzelt Erwähnung gefunden. Eine Ausnahme stellt die Kommentierung von Cramer/Perron, in: Schönke/ Schröder, 28. Aufl. 2010, zu $₫ 263$ StGB dar, wo die Dissertation mehrfach als zitierwürdig erachtet worden ist (z.B. Rdnrn. 16c, 39) und damit ihre Berechtigung erfahren hat. Dies nicht zu unrecht angesichts des Umstandes, dass die im Untertitel angesprochene „,formale Betrachtungsweise“ des BGH zur Schadensermittlung bei Betrugsdelikten im kassen(vertrags-)ärztlichen Bereich von entscheidender Bedeutung ist.

Luig betrachtet in der von Kühne (Fachbereich Rechtswissenschaften der Universität Trier) betreuten Dissertation zunächst das System der vertragsärztlichen Versorgung und zeichnet sich hier durch erstaunliche Sachkunde aus - nicht zuletzt wohl beeinflusst durch seinen Vater, der als Chefarzt wertvolle Hinweise aus der Praxis gegeben haben wird. Nach einer Übersicht zu den Grundlagen der Abrechnung kommt Luig zu den Anwendungsfällen der streng formalen Betrachtungsweise des BGH (auf der Grundlage des Urt. v. 28.9.1994, NStZ 1995, 85, 86) mit Hinweisen zur Rechtsprechung bezüglich der Delegation, der verdeckten Angestelltenverhältnisse und des ,,Strohmanns“ (in Zusammenhang mit „Budgeterhöhungen) und unterzieht diese Konstellationen einer rechtlichen Würdigung im Hinblick auf $§ 263$ StGB. Dabei wird der Irrtumsproblematik im Verhältnis zu den

Rechtsanwalt Prof. Dr. iur. Franz-Josef Dahm,

Essen, Deutschland
Erkenntnismöglichkeiten einer KV als Abrechnungsstelle besondere Aufmerksamkeit gewidmet.

Im zweiten Teil der Arbeit (ab S. 97) befasst sich Luig mit Einzelheiten der Schadensproblematik und der ,,streng formalen Betrachtungsweise" und zeigt unterschiedliche Lösungsansätze für die in Betracht kommenden Konstellationen auf. Hierbei legt er den herrschenden juristisch-ökonomischen Vermögensbegriff unter Berücksichtigung eines objektiv-individuellen Schadenseinschlags zugrunde. Entscheidend ist nach Luig der Vergleich der Vermögenslage des mutmaßlich Geschädigten vor und nach der Vermögensverfügung, der entsprechend dem Schutzzweck der Norm nach dem Leistungsempfänger (KV/Krankenkasse oder Patient) individualisiert wird.

Im Schlussteil beleuchtet Luig Detailprobleme des Abrechnungsbetrugs bezogen auf einzelne Fallgruppen. Hervorgehoben werden soll hier nur die Konstellation der ,,verkappten Gemeinschaftspraxis“ (dazu zuletzt BSG, Urt. v. 23.6.2010 - B 6 KA 7/09 R -), für deren Abrechnung Luig den Tatbestand des $\$ 263$ StGB verneint wissen will. Hierbei wird übersehen, dass jedenfalls für zurückliegende Sachverhaltsgestaltungen vor dem 31.12.2006 (Einführung des \$98 Abs. 2 Nr. 13 SGB V mit erweiterten Möglichkeiten der Anstellung, dazu Dahm/Schmidt [2007], in: Rieger/Dahm/Steinhilper [Hrsg.], HK-AKM, Nr. 1780 „Falschabrechnung/Abrechnungsbetrug“, Rdnrn. 42, 44) ein Betrug schon deshalb zwingend anzunehmen war, weil mit der Zulassung der ,verkappten Gemeinschaftspraxis“ die Möglichkeit eröffnet wurde, etwa den um $10 \%$ erhöhten „Ordinationskomplex“ zu beanspruchen (Einzelheiten bei Dahm/Schmidt, a.a. O., Rdnr. 44).

Ganz unabhängig davon, ob man Luig (wie etwa Cramer/Perron, a.a. O.) in allen von ihm besprochenen ,Schadendetails" folgen will, handelt es sich um eine bemerkenswerte Dissertation, die bei der Diskussion um Probleme des Abrechnungsbetrugs künftig nicht übergangen werden kann. Dies schon deswegen, weil sie sich durch eine Detailkenntnis im Abrechnungswesen auszeichnet, die vielfach im strafrechtlichen Schrifttum zu vermissen ist.

Abgerundet wird die gelungene Dissertation durch ein ausführliches Literaturverzeichnis, welches weitgehend den Stand der Literatur bis zum 30.6.2009 widerspiegelt. Die Dissertation ist jedem mit der Frage des Abrechnungsbetruges Befassten zu empfehlen. 